\title{
Seguimiento de Niños con Peso de Nacimiento Inferior A $1.500 \mathrm{~g}$.
}

\author{
Dra, Elizabeth Weldt S. ${ }_{i}$ Dr. Fnrique Hering A.1; Dr. Bolívar Valenzuela M. ${ }^{2}$; \\ Psic, Gloria Angulo M.1: Matr. Ana Neira G. ${ }^{1}$.
}

Follow Up of Infants with Brith Weight Under $1.500 \mathrm{~g}$.

\begin{abstract}
Infants with birth weight less than $1.500 \mathrm{~g}$ born between July 1981 and December 1983 were lollowed up for periods of 1 to 3 years. Ninetyone infants, discharged from the Neonatal Intensive Care Unit were included at the start of the study. Eighteen $(19.8 \%)$ were lost to follow up and two died in the first year of life. During the follow up period, 35 of them $(49.29 \%)$ were admitted to a Children's Hospital because of respiratory or developmental problems. Eleven patients (15.49\%) had neurologic sequelae, which consisted in cerebral palsy in nine and seizures in other two infants. Seven of the nine children which eventually showcd evidence of cerebral palsy had evidence of intracraneal hemorrhage in the neonatal period. Visual disturbances were cvidenced in 4 children, two of them with signs of retrolental fibroplasis. Using the corrected age of gestation, the growth pattern of these patients was placed between the 10th and the 50th percentile.

(Key words: Very low birth weigh. Prcmaturity. Follow up. Secuelae).
\end{abstract}

Los niños con peso de nacimiento inferjor a $1.500 \mathrm{~g}$ han constituido un grupo con elevada Morbilidad y Mortalidad, que tiende a disminuir en relación a los avances de los cuidados perinatales ${ }^{1-2-3}$.

En la Maternidad del Salvador, en el año 1982, de un total de 11.420 Recién Nacidos vivos, $1.06 \%$ tuvo un peso inferior a $1.500 \mathrm{~g}^{4}$. En este grupo, la sobrevida fue de $38.8 \%$, elevándose a $43.8 \%$ en 1983 . Se debe destacar la sobrevida de $9.3 \%$ de Recién Nacidos de menos de 750 g en 1983 versus $0 \%$ en 1982 .

La recuperación de los niños de muy bajo peso, que en general concentran la mayor y más compleja Morbilidad en el período neonatal, hace que sea del más alto interés poder conocer la calidad de su sobrevidà y la posibilidad de aparición de secuelas a largo plazo. Este ha sido el motivo para efectuar un estudio prospectivo en 91 Recién Nacidos dados de alta de la Unidad de Neonatologia de la Maternidad del Salvador.

1. Unidad de Neonatologia, Maternidad del Hospital del Salvador. Servicjo de Salud Metropolitano Oriente.

2. Servicio Neurología, Hospital Luis Cavo Mackenna. Servicio de Salud Metropolitano Oriente.
Los objetivos del presente trabajo fueron: estudiar y conocer el desarrolio antropométrico y psicomotor de niños con peso de nacimiento inferior a $1.500 \mathrm{~g}$, estudiar su morbilidad y en el grupo con secuelas, analizar sus posibles etiologías. Se consideraron para el estudio aquellos niños con seguimiento superior a un año.

\section{MATERIAL Y METODO}

El grupo estuvo constituido por 91 niños nacidos entre Julio de 1981 y Diciembre de 1983 , con un peso de nacimiento inferior a $1.500 \mathrm{~g}$, que fueron dados de alta de la Unidad de Neonatologia de la Maternidad del Salvador.

La distribución por peso y año de nacimiento de los nifnos en control se muestra en la Tabla $l$., destacando un mayor número de nixos nacidos en 1983,10 de los cuales pesaron menos de $1.000 \mathrm{~g}$ al nacer. La distribución por sexo fue muy similar: $44 \%$ sexo masculino y $56 \%$ sexo femenino.

El peso era adecuado para la edad gestacional en 67 casos. Hubo 24 recién riacidos pequeños para la edad gestacional, que tuvieron como antecedente más frecuente el de hipertensión materna $(60 \%)$.

Todos los recién nacidos con algunas manifestaciones de vida ingresaron a la Unidad de Tra- 
Tabla 1 .

Distribución por Peso de Nacimiento de 91 Recién Nacidos

\begin{tabular}{ccccc}
\hline Año Nacimiento: & -1000 & $1001-1250$ & $1251-1500$ & Total \\
\hline 1981 & 1 & 7 & 12 & 20 \\
1982 & 1 & 8 & 16 & 25 \\
1983 & 10 & 14 & 22 & 46 \\
\hline Total & 12 & 29 & 50 & 91 \\
\hline
\end{tabular}

tamiento Intensivo Neonatal, donde recibieron el tratamiento necesario.

En la Tabla 2, se muestra la morbilidad que presentaton en el período neonatal los 91 niños incluidos en el seguimiento. De los 25 que sufrieron por sindrome de dificultad respiratoria, 10 requirieron ventilación mecánica. En 17 con hemorragias intracraneanas demostradas, mediante ecografía encefálica, hubo 5 hemorragias subependimarias; 6 hemorragias intraventriculares sin dilación ventricular; 3 hemorragias intraventiculares con dilación ventricular; 2 hemorragias intraventriculares con extensión paren. quimatosa y una hemorragia de fosa posterior. Los casos de apnea, tanto primarias y secundarias, constituyeron un problema frecuente. Las infecciones mayores que correspondieron a 5 bronconeumonias, 3 septicemias, 2 infecciones del tracto urinario y 1 meningitis.

Tabla 2.

Morbilidad en el Período Neonatal de 91 Recién Nacidos con Peso de Nacimiento Inferior a $1.500 \mathrm{~g}$

Síndrome de Diticultad Respiratoria:

Hemorragia Intracianeana:

25

Apneas Primarias:

Apneas Secundarias:

Infecciones Mayores:

En una entrevista previa al alta, los niños fueron citados a Policlínico de seguimiento de alto riesgo neonatal. El control fue realizado por pediatra y psicólogo, cada 3 meses en el primer año de vida, cada 6 meses en el segundo y luego una vez af año. Este control se hizo más a menudo cuando las condiciones clínicas lo requerían. En cada uno se consignaron las cifras antropométricas y se efectuó medición del đesarrollo psicomotor utilizando la escala de evaluación de 0 a 2 años $y$ la Prueba de Griffiths.

Al aflo de edad los pacientes fueron evaluados por neurólogo infantil y un examen of talmológico se solicitó en aquellos niños en_que clinicamente, se sospecharon fallas de la visión.
En los diferentes controles, las medidas antropométricas $y$ el desarrollo psicomotor fueron corregidos restándoles a la edad cronológica, el tiempo que faltó para completar las 40 semanas de gestación, lo que se hizo hasta la edad de 3 años 2,3 .

De los niños que iniciaron el control, 18 abandonaron el seguimiento $(19,8 \%)$ por incum. plimiento de las citaciones y dos niños fallecieron, uno a los tres meses de edad por broncoaspiración de leche: este lactante tenía el antecedente de hemorragia intracraneana en el periodo neonatal y su peso de nacimiento fue $1.495 \mathrm{~g}$. El otro caso correspondió a un niño con peso de nacimiento de $1.120 \mathrm{~g}$ que presentó apneas secundarias a hemorragia intracraneana, ja Tomografía Axial Computarizada demostró malformaciones múltiples lo que determinó su deceso a los 4 meses de edad.

De los restantes 71 niños, 17 han completado 3 años de control; 14 casos 2 años y 40 entre 1 y 2 af̂́os.

\section{RESULTADOS}

La Tabla 3 muestra que durante el primer año de vida, 22 nifios $(30,9 \%)$ sufrieron problemas respiratorios agudos, de los cuales $14(63,6 \%)$ requirieron hospitalización por esta causa.

En 21 niños fue necesario realizar tratamientos quirúrgicos por hernia inguinal y en 14 lactantes se encontró anemia ferropriva a pesar de habérseles indicado fierro en forma preventiva.

Tabt 3.

Morbilidad Detectada en el Primet Año de Vida en 71 Niños en Control con Peso de Naciniento Inferior a $1.500 \mathrm{~g}$.

Problemas Pulmonares Agudos: 
Las alteraciones de vision se encontraron en 4 nifios; en 2 había Fibroplasia Retrolental $(850 \mathrm{~g}$ a $1.200 \mathrm{~g}$ de peso al nacer, Hemorragia Intracraneana e indicación de varias transfusiones). En 1 caso se diagnosticó estrabismo $(1.20 \mathrm{~g})$ y en otro estrabismo y miopia $(1.240 \mathrm{~g})$. En 11 nínos $(15,49 \%)$ se detectaron problemas neurológicos que se detallan en Tabla 4.

De los 1 I niños controlados, que desarrollaron problemas neurológicos en el primer año de vida, 9 correspondieron a parálisis cerebral. Como se puede apreciar, todos son recién nacidos de muy bajo peso $y$, en 7 la ecografía permitió demostrar una hemorragia intracraneana.

En los 2 casos restantes no se realizó ecografía, motivo por el cual no se pudo definir la etiologia del proceso.

Los niños que presentaron convulsiones en el transcurso del seguimiento, no tuvieron manifestaciones neurológicas en el período neonatal y por lo tanto no se efectuó en ellos ecografía encefálica.

En la Figura I se observa el crecimiento de las niñas en relación a peso, talla y circunferencia de cráneo con edad corregida según edad gestacional. Aquetlas nirfas que al nacer tuvieron un peso adecuado para la edad gestacional, mantuvieron el peso entre percentil 10 y 25 y la talla y circunferenciá de cráneo estuvieron más cercanas a la media.

Aquellas niñas que fueron pequeñas para la edad gestacional recuperaron más tardíamente la curva normal de crecimiento.

La diferencia entre estos dos grupos fue estadísticamente significativa en peso y talla sólo el primer mes de edad corregida ( $p<0.001)$.

Los niños de sexo masculino siguen una curva muy similar a lo observado en la Figura 1.

Cabe destacar que, utilizando las curvas de crecimiento según edad gestacional corregida, sóto 5 niños estaban bajo el percentil 10 en peso, talla y circunferencia de cráneo. Dos de estos niños son portadores de parálisis cerebral.

El desarrollo psicomotor fue adecuado para la edad corregida, excepto en 9 niños que presentaron parálisis cerebral como secuela neurológica.

\section{DISCUSION}

El seguimiento en largo plazo de los Recièn Nacidos de Muy Bajo Peso es de gran importancia, pues penmite conocer la calidad de sobrevida de ellos, pesquisar precozmente algunas secuelas e iniciar su rehabilitación oportunamente.

El grupo estudiado es de alto riesgo de morbimortalidad en el periodo neonatal inmediato $y$

Tabla 4.

Trastornos Neurológicos en relación con el Peso de Nacimiento y la Lesión Neurológica del Período Neonatal en 11 niños con Peso đe Nacimiento inferior a 1.500 grs.

\begin{tabular}{|c|c|c|}
\hline PROBLEMAS NELROLOGICOS & $\begin{array}{l}\text { P. NAC. } \\
(\mathrm{g})\end{array}$ & ECOGRAFIA ENCEFALICA \\
\hline \multicolumn{3}{|l|}{ I. PARALISIS CEREBRAL } \\
\hline \multirow{4}{*}{$\begin{array}{l}\text { Paraparesia } \\
\text { cerebral } \\
\text { espástica (4 casos) }\end{array}$} & 850 & Hemorragia Subependimaria \\
\hline & 930 & Hemorragia Ventricular con dilatación Ventricular \\
\hline & 1.100 & Hemorragia Ventricular con dilatación Ventricular \\
\hline & 1.220 & $-\cdot-----------$ \\
\hline \multirow{4}{*}{$\begin{array}{l}\text { Hemiparesia } \\
\text { cerebral } \\
\text { espástica ( } 4 \text { casos) }\end{array}$} & 700 & Hemorragia Ventricular con extensión parenquimatosa \\
\hline & & Hemorrsaria Yantrimur contención namanimatoca \\
\hline & 930 & Hemorragla ventricular con extension parenqumatosa \\
\hline & $\begin{array}{l}1.200 \\
1.430\end{array}$ & Hemorragia Ventricular sin dilatacion Ventricular \\
\hline \multicolumn{3}{|l|}{$\begin{array}{l}\text { Diplejia cerebral } \\
\text { espástica con retardo }\end{array}$} \\
\hline mental (1 caso) & 1.200 & Hemorragia Ventricular con dilatación Ventricular \\
\hline \multicolumn{3}{|l|}{ II. CONVULSIONES } \\
\hline \multicolumn{3}{|l|}{ Crisis comicia única } \\
\hline (1 caso) & 1.410 & ------------ \\
\hline \multicolumn{3}{|l|}{ Crisis convulsivas } \\
\hline repetidas (1 caso) & 1.400 & ------------ \\
\hline
\end{tabular}



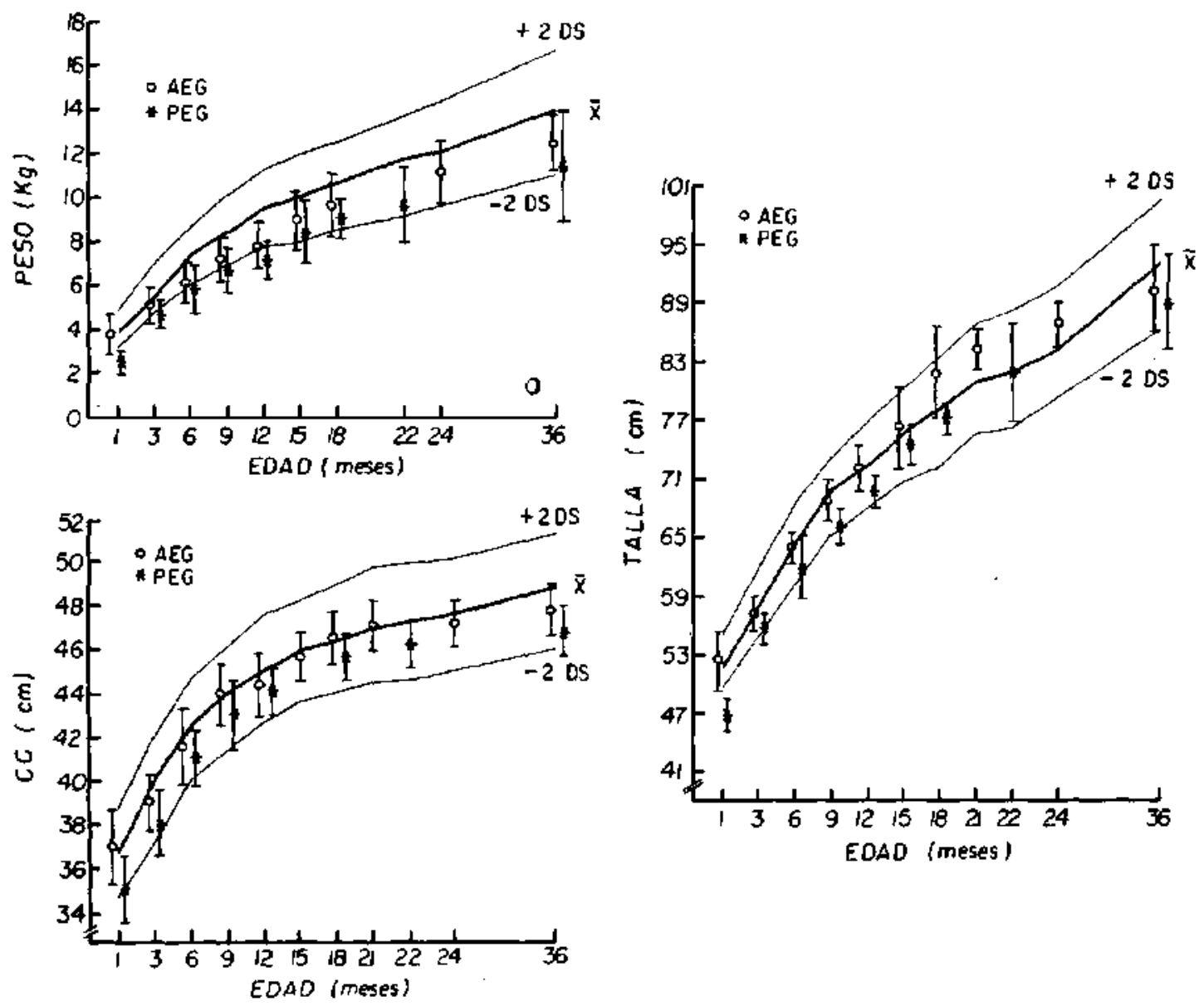

Figura 1: Medida de Peso, Tảla y Circunferencín de Cráneo (CC) de 30 niñas Adecuadas y 15 niñas Pequeñas para la Edad Gestacional (AEG y PEG) Edad corregida según gestación entre 1 y 36 meses (Curva de Antropometría del Niño Chileno de 0 a 6 años, de Alfredo Patri y Cols.).

en el transcurso del primer año de vida. Kulkar$n i^{6}$ describió una incidencia de 3 a $4 \%$ de mortalidad en este periodo, mostrando que en la mitad de los casos ésta se debe a defectos neurológicos o enfermedades respiratorias agudas, y en otra mitad, a muertes súbitas que ocurren entre el primer y tercer mes de vida. En nuestra casuística se comprobó el fallecimiento de dos niños del total del grupo (2,79\%). Sin embargo, queda la interrogante de la exactitud de dicha cifra, puesto que el porcentaje de abandono a control fue alto $(19,8 \%)$.

En nuestro país, las Unioades de Cuidado Intensivo Neonatal han significado mayor sobrevida de niños de muy bajo peso. Esto hace recomendable que existan, en cada Unidad, facilidades necesarias para poder estudiar la calidad de la sobrevida, con la integración de distintos especialistas y en estrecha conexión con el nivel de atención primaria. La integración es necesaria por la alta frecuencia con que estos niflos requieren hospitalizaciones, ya sea por alteraciones del crecimiento $y$ desarrollo, $o$ por infecciones. Mc--Cornick ${ }^{7}$, da una cifra de $40 \%$ de hospita: lizaciones en el primer año de vida. En nuestro grupo, las hospitalizaciones por enfermedades agudas y quirúrgicas alcanzaron a $49,29 \%$.

En lo referente a alteraciones de visión, repor. tes extranjeras $8,9,10,11$ destacan que la mayor incidencia de ellas se presenta en el recién nacido prematuro menor de $1.000 \mathrm{~g}$, en los cuales se ha descrito 38 y $58 \%$ de Retinopatía del prematuro, con 22 a $42 \%$ de lesiones cicatriciales y 5 a $11 \%$ de ceguera; entre los reciên nacidos de 1.001 a $1.500 \mathrm{~g}$, sólo $2,2 \%$ desarrollan secuelas cicatriciales $y$ de 0,3 a $1 \%$ son ciegos. El númerö de niños ciegos depende de la incidencia de retinopatía del prematuro en fase cicatricial, del núme- 
ro de niños en riesgo de presentar enfermedad y de la mortalidad del grupo.

Entre nuestros pacientes, se encontraron 2 con Fibroplasia Retrolental en el examen oftalmológico dirigido. La verdadera incidencia deberá estudiarse en forma prospectiva, siendo probable es que nuestros porcentajes sean inferiores a los publicados en series extranjeras, debido a que la mayor incidencia de este proceso ocurre en niños de peso muy bajo al nacer, que en nuestro medio tienen todavía una alta tasa de mortalidad neonatal.

Otro aspecto importante en el seguimiento de los niffos de bajo peso es la incidencia de secuelas neurológicas, observándose relaciones entre he. morragia intracraneana y parálisis cerebral. El númeto de niños en control es aún pequeño, y el tiempo de seguimiento corto, para obtener datos definitivos, pero las secuelas neurológicas graves se diagnostican antes del año de edad quedando los problemas de aprendizaje para el futuro próximo ${ }^{2}$.

El crecimiento pondoestatural fue el esperado, ${ }^{2,35}$ destacando el atraso en recuperar parámetros normales de los niños catalogados como "Pequeños para la Edad Gestacional", en el momento de nacer. Es importante resaltar que para poder efectuar comparaciones y una evaha. ción correcta del estado de nutrición, es recomendable corregir la edad post-natal de acuerdo a edad gestacional y usar las curvas de niños normales. Se debe tomar en consideración que la curva ponderal es influida por enfermedades graves durante el período neonatal, hipoalimentación $\mathrm{y}$ ayuno en los primeros días de vida.

La mejor atención perinatal y la formación de Unidades de Cuidado Intensivo Neonatal, han logrado aumentar la sobrevida de los recién nacidos de bajo peso y al mismo tiempo permitido mejorar su pronóstico en el largo plazo $2,15,16$. Aún cuando la incidencia de secuelas es relativamente baja, son indispensables los seguimientos multidisciplinarios para diagnosticarlas y tratarlas precozmente reduciendo sus efectos y buscando sobrevidas similares a las de los niños con peso normal al nacer.

El medio familiar tiene gran influencia en el desartollo de la personalidad y la inteligencia del niño ${ }^{17}$, por esto, es necesario integrar a la familia en el manejo del recién nacido de bajo peso, to que comienza con el ingreso de los padres a las Unidades de Neonatología y continúa durante los controles posteriores donde se debe indicar estimulación psicomotora e informar sobre los problemas habituales de los niños de bajo peso al nacer. Entre estos problemas destacan la aparente lentitud del crecimiento y desarnollo en relación a la edad cronológica, en circunstancias que la evaluación debe ser efectuada en relación a edad corregida según duración de la gestación.

Creemos al igual que Beca y cols. ${ }^{18}$, que el bajo peșo de nacimiento, por sí solo, no influye en el pronóstico neurológico y que serían los factores perinatales relacionados con hipoxia, hemorragia intracraneana, hipoglicemias e infec. ciones del sistema nervioso central los responsa. bles de éril ${ }^{9}$.

\section{RESUMEN}

Se estudiaron 91 nif̃os con peso de nacimien to inferior a 1.500 grs., nacidos en la Maternidad del Salvador y controlados en el policlínico de seguimiento de alto riesgo neonatal. El $19,8 \%$ de los pacientes abandonó el control y 2 niños fallecieron en el primer año de vida.

El grupo quedó constituido por 12 niños con peso de nacimiento inferior a $1.000 \mathrm{~g}, 29$ que pesaron entre 1.001 y $1.250 \mathrm{~g}$ y 50 cuyo peso fue superior a $1.251 \mathrm{~g}$. Se comprobó que $23 \%$ necesitaron reparación quirúrgica de hernias inguinales; en 14 niños (15,38\%) se diagnosticó anemia ferropriva, a pesar del uso preventivo de fierro, y $15,38 \%$ de los pacientes fueron hospitalizados en algún momento del primer año de vida por problemas agudos en su mayoría respira. torios. En 4 niños se demostraron alteraciones de la visión, que en 2 casos correspondian a fibroplasia retrolental. Nueve pacientes desarrollaron evidencia de parálisis cerebral $(12,67 \%)$, en 7 existían antecedentes de hemorragia intracraneana. El desarrollo pondoestatural se mantuvo dentro de los rangos normales de crecimiento en los niños que fueron adecuados para la Edad Gestacional, no así en los pequeños para la edad gestacional.

\section{REFERENCIAS}

1. Koops. B., Morgan. L., Battaglia. F.: Neonatal Mortality risk in relation to birth weight and gestational age: Update. J. Pediatr. 101: 969, 1982.

2. Hirata. T., Epcar. J., Walsh. A.: Survival and outcome of $501-750 \mathrm{~m}$. a six year experience. J. Pediatr, 102: 741, 1983.

3. Bennett. F., Robinson. N., Sells, C.: Crecimiento y Desarjollo de los niños con peso de nacimiento inferior a $800 \mathrm{~g}$. Pediatrics, 15: 174, 1983. (Edición Española).

4. Weldt, E., Alarcón, J., Vaisman. S.: Morbilidad y Mortalidad de Recién Nacidos con peso de Nacimiento inferior a 1.500 g. Rev. Chil. Pediatr. 5S: 327,1984 . 
5. Rodriguez, S., Arancibia. V., Undurraga. C.: Escala de Evaluación del Desarrollo Psicomotor de 0 a 24 meses Editorial Galdoc 2a. Edición 1983.

6. Kulkarni. P., Hall. R., Rhodes. $P$ et ol: Postneona. tal Infant Mortality in Infants Admitted to a Neonatal Intensive Care Unit. Pediatrics 62: 178, 1978.

7. Mc-Cornick. M., Shapiro. S., Starfield. B.: Rehospitalization in the first year of like for High-Risk Surviyors. Pediatrics 66: 991, 1980.

8. Phelps. D.: Retinopathy of Prematurity. An estimate of Vision Loss in the United States 1979. Pediatrics 67: 924, 1981.

9. Teplin. S.: Development of Blind Infants and Children with Retrolental libroplasia: implications for physician. Pediatrics 71: 6, 1983.

10. Johnson. L., Shceffer. D., Goidstein. D., et al.: Prediction of Retrolental Ftbroplasia (RLF). - Pediatr. Res. 15: 665, 1981.

11. Shohat. M., Reisuer. S., Krikier. R.: Retinopatía de la prematuridad: incidencia $y$ factores de riesgo. Pediatrics Edición Española 19: 102, 1983.

12. Sagal, S., Rosembaum. P., Sroskopf. B., et aL: Follow up of infants 501 to $1.500 \mathrm{~g}$ birth weight delivered to residents of a geographically defined region with perinatal intensive care. J. Pediatr. 100: 606,1982 .
33. Sinclair. J., Torrance. G, Boyle. M., et el.: Evahuation of Neonatal Intensive Care. N. Engl. J. Med. 305. 489, 1981 .

14. Pape, $K_{+}$, Buncic. $J ., A$ shby. $S$, et al: The Status at two years of low birth weight infants born in 1974 , with birth weights of less than $1.001 \mathrm{~g}$. J. Pediatr. 92: $253,1978$.

15. Kimble. K., Ariagno. R., Stevenson. D., et al.: Growth to age 3 ycars among very low birth weight sequelae free survivors of modern neoratal intensive care. J. Pediatr, 100: 622, 1982.

16. Scewart. A., Turcan, D., Rawlings. G., et al.* Prognosis for infants weighing 100 or less at birth. Arch. Dis. Child. 52: 97, 1977.

17. Montenegro. H., Lira. M., Rodríguez. S, y cols.: Programa de estimulación precoz, informe final. Ministerio de Salud. Santiago Chile 1976.

18. Beca. J.P., Román. C., Zubaty. L., Abourbih. J.: Estudio de desarrollo de nin̄os considerados de alto riesgo neurológico en el período neonatal. Rev." Chil. Pediats. 51: 97, 1980.

19. Mac Donald. M., Koops. B., Johnson. M.: Cronología y antecedentes de $\mathbf{k}$ Hemorragia Intracraneal en el Recién Nacido. Pediatrics. Edic. Española. 18: 14, 1984. 